\title{
Large Magellanic Cloud Distance from Cepheid Variables using Least Squares Solutions
}

\author{
C. Ngeow ${ }^{1}$ and S. M. Kanbur ${ }^{2}$ \\ 1 University of Illinois, Urbana, IL 61801, USA cngeow@astro.uiuc.edu \\ 2 State University of New York at Oswego, Oswego, NY 13126, USA \\ kanbur@oswego.edu
}

Distance to the Large Magellanic Cloud (LMC) is determined using the Cepheid variables in the LMC. We combine the individual LMC Cepheid distances obtained from the infrared surface brightness method and a dataset with a large number of LMC Cepheids. Using the standard least squares method, the LMC distance modulus can be found from the ZP offsets of these two samples. We have adopted both a linear P-L relation and a "broken" P-L relation in our calculations. The resulting LMC distance moduli are $18.48 \pm 0.03 \mathrm{mag}$ and $18.49 \pm 0.04 \mathrm{mag}$ (random error only), respectively, which are consistent to the adopted 18.50 mag in the literature.

\section{Introduction}

Recently, [1] (hereafter G05) has used the infrared surface brightness method to obtain the individual distances to 13 LMC Cepheids with an averaged LMC distance modulus of $18.56 \pm 0.04 \mathrm{mag}$ (random error only). However, LMC hosts more than 600 Cepheids with data available from the Optical Gravitational Lensing Experiment (OGLE) [2]. A linear least squares solution (LSQ) will allow a simultaneous determination for both of the LMC distance and the P-L relation (see Figure 1).

\section{Data, Method and Results}

The data include the absolute magnitudes for the 13 Cepheids with individual distance measurements from G05 and the apparent magnitudes (after extinction correction) for $\sim 630$ LMC Cepheids from [3], which is based on the OGLE database. For both datasets, we fit the following regression using the LSQ: $x=\alpha \Delta \mu+a+b \log (P)$ where $\alpha=0$ if $x=M$ (for G05 data) or $\alpha=1$ if $x=m$ (for OGLE data). The results of the LSQ are: $M^{V}=-2.76 \pm 0.04 \log (P)-1.36 \pm 0.07$ with $\Delta \mu(V)=18.47 \pm 0.06$, and 


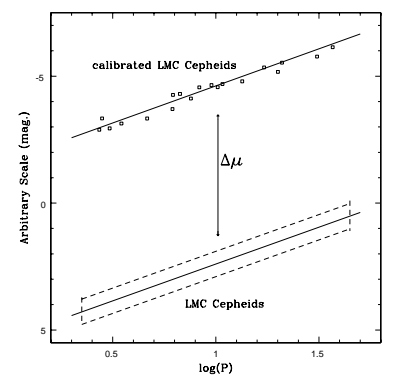

Fig. 1. Illustration of the LSQ. Introducing an offset, $\Delta \mu$, between the calibrated Cepheids and the other LMC Cepheids will allow us to simultaneously solve for the LMC distance modulus and the P-L relation with LSQ.

$M^{I}=-2.98 \pm 0.02 \log (P)-1.86 \pm 0.05$ with $\Delta \mu(I)=18.48 \pm 0.04$. The weighted average of these $\Delta \mu$ is $18.48 \pm 0.03 \mathrm{mag}$ (random error only).

Since the recent studies have strongly suggested the LMC P-L relation is not linear $([3,4,5,6])$, we also use the following "broken" regression to fit the data:

$$
x=\alpha \Delta \mu+a_{S}+\beta b_{S} \log (P)+\gamma\left(a_{L}-a_{S}\right)+\epsilon b_{L} \log (P), \alpha=\left\{\begin{array}{l}
0 \text { if } x=M \\
1 \text { if } x=m
\end{array}\right.
$$

where subscripts $S_{S}$ and $L$ refer to the short and long period Cepheids, respectively, and $\beta=1, \gamma=0, \epsilon=0$, for $\log (P)<1.0 ; \beta=0, \gamma=1, \epsilon=$ 1 , for $\log (P) \geq 1.0$. The results are: $M_{L}^{V}=-2.84 \pm 0.16 \log (P)-1.23 \pm$ $0.21 ; M_{S}^{V}=-2.94 \pm 0.06 \log (P)-1.28 \pm 0.07$ with $\Delta \mu(V)=18.49 \pm 0.06$, and $M_{L}^{I}=-3.09 \pm 0.11 \log (P)-1.69 \pm 0.14 ; M_{S}^{I}=-3.09 \pm 0.04 \log (P)-1.80 \pm 0.05$ with $\Delta \mu(I)=18.49 \pm 0.04$. The weighted average of the distance moduli in both bands is $18.49 \pm 0.04 \mathrm{mag}$ (random error only). F-test $([4,5])$ is also applied to examine if the data is more consistent with a single-line regression (the null hypothesis) or a two-lines regression (the alternate hypothesis). For our data, we obtain $F(V)=7.3$ and $F(I)=6.9$, where $F \sim 3$ at $95 \%$ confident level. This suggested the null hypothesis can be rejected and the data is more consistent with the broken P-L relation.

\section{References}

1. Gieren, W., et al., 2005, ApJ, 627, 224 (G05)

2. Udaski, A., et al., 1999, Acta Astron., 49, 223

3. Kanbur, S. \& Ngeow, C., 2006, MNRAS, 369, 705

4. Kanbur, S. \& Ngeow, C., 2004, MNRAS, 350, 962

5. Ngeow, C., et al., 2005, MNRAS, 363, 831

6. Sandage, A., Tammann, G. A. \& Reindl, B., 2004, A\&A, 424, 43 